Cultures \& Conflits

42 | été 2001

Le crime organisé en Russie : nouvelles approches

\title{
Le crime organisé en Russie: un état des lieux à
}

\section{Saint-Pétersbourg}

lakov Kostioukovski

\section{(2) OpenEdition}

1 Journals

\section{Édition électronique}

URL : http://journals.openedition.org/conflits/557

DOI : $10.4000 /$ conflits.557

ISSN : $1777-5345$

Éditeur :

CCLS - Centre d'études sur les conflits lilberté et sécurité, L'Harmattan

Édition imprimée

Date de publication : 1 juin 2001

ISBN : 2-7475-1117-0

ISSN : 1157-996X

\section{Référence électronique}

lakov Kostioukovski, « Le crime organisé en Russie: un état des lieux à Saint-Pétersbourg », Cultures \& Conflits [En ligne], 42 I été 2001, mis en ligne le 20 mars 2006, consulté le 30 mars 2021. URL : http:// journals.openedition.org/conflits/557 ; DOI : https://doi.org/10.4000/conflits.557

Ce document a été généré automatiquement le 30 mars 2021.

Creative Commons License 


\title{
Le crime organisé en Russie: un état des lieux à Saint-Pétersbourg
}

\author{
lakov Kostioukovski
}

De nombreux mythes sont fréquemment associés au thème de la criminalité, que ce soit par des politiciens à des fins populistes ou par des journalistes en quête de sensations. Le crime organisé se prête particulièrement à ces usages. Or, alors que ce phénomène est étudié et débattu depuis les années 1920 dans des pays «capitalistes» tels que l'Italie, les Etats-Unis ou le Japon, il représente une nouveauté dans le contexte russe post-soviétique. En 1986, le discours scientifique soviétique sur le crime organisé se limitait à des interrogations sur son éventuelle existence en U.R.S.S. Au cours des années 1990, de nombreux travaux scientifiques ${ }^{1}$ et investigations journalistiques ${ }^{2}$ ont été consacrés à ce sujet. Pour autant, la connaissance du crime organisé en Russie demeure insuffisante. Les fondements méthodologiques restent imprécis et les travaux russes sont souvent déconnectés des recherches menées ailleurs sur ce sujet ${ }^{3}$.

Le département de sociologie de la déviance et du contrôle social (le centre d'étude de la déviance) de la filiale peterbourgeoise de l'Institut de sociologie de l'Académie des sciences de Russie a mené en 1993 une première étude sur le crime organisé, qui a motivé la création, à partir de 1995, d'un axe permanent de recherches ${ }^{4}$. L'objectif de cet article est de présenter ces travaux et de décrire l'évolution du crime organisé au niveau local, dans la ville de Saint-Petersbourg.

Fondements théoriques et méthodologie

Les criminologues considèrent le crime comme un phénomène social complexe, défini selon deux critères : le danger qu'il représente pour la société (critère dit objectif) et la manière dont il est pris en compte dans la législation pénale (critère dit subjectif). Il n'existe pas de crimes ou d'infractions per se. La criminalité est un construit social, relatif (qui varie dans le temps et l'espace) et conventionnel (qui est un produit de la législation) ${ }^{5}$. La délinquance d'affaires illustre parfaitement le caractère relatif et mouvant des frontières entre le licite et l'illicite.

Trois approches ont pu être appliquées à la criminalité organisée, en tant que forme spécifique de criminalité. Le caractère ethnique de certaines organisations criminelles 
a pu motiver des recherches anthropologiques ${ }^{6}$. D'autres travaux ont privilégié l'aspect organisationnel de cette forme de délinquance et essayé de comparer le fonctionnement interne des organisations criminelles à celui des bureaucraties ou des grandes firmes, et leurs relations avec l'environnement extérieur à des relations patrimoniales ou clientélistes ${ }^{7}$. Enfin, la criminalité organisée a pu être considérée comme une entreprise criminelle (business enterprise), notamment quant à la stratégie de développement des différentes activités menées ${ }^{8}$. Ces trois approches peuvent être combinées, mais, dans notre perspective, le concept d'entreprise criminelle s'avère le plus fécond pour l'analyse. Les organisations criminelles poursuivent des objectifs qui sont ceux d'une entreprise criminelle; elles les réalisent en étant parfois fondées sur une base ethnique et en étant toujours structurées selon un principe hiérarchique.

Pour nous, la criminologie est d'abord une sociologie du crime. La criminalité organisée peut être étudiée en utilisant les sources et méthodes habituellement mobilisées dans les sciences sociales: analyse socio-historique comparée afin de repérer les traits caractéristiques de l'évolution des organisations criminelles, analyse des textes officiels et des documents normatifs, des dossiers judiciaires, des données statistiques, des publications journalistiques. Des entretiens peuvent être menés avec des représentants des services répressifs, des entrepreneurs, des banquiers en contact dans leur vie professionnelle avec le crime organisé, voire des membres des organisations criminelles. Il est enfin possible d'interroger la population au moyen de questionnaires sur les représentations relatives au crime organisé dans la société. Toute forme d'observation du milieu criminel demeure cependant dangereuse et extrêmement limitée.

Dans le cadre de cet article, nous exploitons les entretiens que nous menons depuis 1994 avec des membres du milieu criminel. Nous disposons de 270 entretiens, qui ont été soit enregistrés, soit retranscrits de mémoire immédiatement après leur réalisation. Certains de nos interlocuteurs ont accepté de s'entretenir avec nous sur une base régulière (trois fois par an) afin d'évoquer l'évolution de leur activité. Le choix des interlocuteurs s'est initialement opéré à partir de contacts personnels des enquêteurs : un ancien camarade de classe, un voisin, un ami d'un ami qui mène des activités illicites. Par la suite, la méthode de la «boule de neige » a permis de trouver d'autres personnes prêtes à participer à un entretien. L'ensemble des personnes interrogées ne constitue donc pas un échantillon représentatif, mais une agrégation de rencontres fortuites. Nous remarquons cependant que nos interlocuteurs exercent des activités fort variées (fonctions de commandement ou d'exécution, activité générale ou spécialisée). La plupart d'entre eux ont par ailleurs manifesté un réel intérêt pour l'entretien : ils y voyaient l'occasion de réfuter les discours politiques, journalistiques et universitaires sur le crime organisé en présentant eux-mêmes leur activité et leur vision du monde. Les informations recueillies ont toujours été soumises de manière indirecte à d'autres interlocuteurs afin de les vérifier.

En dehors de quatre exceptions, tous nos interlocuteurs sont des hommes. Alors que la grande majorité des simples exécutants est âgée de moins de 25 ans (à l'exception d'anciens combattants ou d'anciens policiers), ceux qui les encadrent sont généralement plus âgés (de 25 à 50 ans). Les interlocuteurs qui se présentent comme des chefs importants n'ont jamais moins de 35 ans. Le niveau d'éducation des interlocuteurs est variable, même si les cas de scolarisation inachevée ou de formation technique sont prédominants. 
Le crime organisé en tant que phénomène social

Le crime organisé représente un phénomène social complexe. Chercher à l'isoler afin de l'analyser relève de la gageure tant il est intriqué dans le tissu social et étroitement lié à d'autres phénomènes sociaux. Par ailleurs, la pertinence de l'expression "crime organisé » est discutable, non seulement parce que toute criminalité est une construction sociale, mais aussi parce qu'une criminalité non organisée ne peut exister. Comme l'a noté V. Ioustitski, "aujourd'hui, alors que l'activité de toute institution publique ou privée est liée à des violations de la législation pénale, la notion de crime organisé est synonyme de celles de société, d'Etat ou d'activité sociale. La notion de crime organisé incarne donc un mal social beaucoup plus général " ${ }^{9}$. L'auteur conclut que, d'un point de vue scientifique, la notion de crime organisé doit être rejetée.

Les définitions du crime organisé sont innombrables. Certaines d'entre elles soulignent les activités menées, d'autres le mode d'organisation des groupes désignés. De manière approximative, on définit habituellement le crime organisé comme "l'activité d'une association de criminels stable et administrée [oupravlaemy], qui commet des infractions sur un mode entrepreneurial et qui se protège du contrôle social par la corruption ». Cette définition figure par exemple dans les documents de la conférence internationale de l'ONU sur le crime organisé en 1991 à Souzdal (Russie) ${ }^{10}$.

De notre point de vue, il faut se garder de comprendre le crime organisé comme un simple ensemble d'activités menées par des organisations criminelles. Il est plus pertinent de le considérer comme un ensemble cohérent d'interactions sociales nouées par une volonté de profit illicite ${ }^{11}$.

Le crime organisé se manifeste avant tout comme une entreprise, une affaire (business) ou une industrie orientée vers la production de biens et/ou de services. L'objectif fondamental du crime organisé est le profit, le bénéfice. De ce point de vue, rien ne le distingue d'une autre entreprise économique. Ce sont les méthodes employées qui lui confèrent une spécificité. Les organisations criminelles accumulent les profits par tous les moyens, notamment criminels. Toute entreprise a cependant recours à des pratiques illicites, voire parfois clairement criminelles, pour atteindre les objectifs fixés, mais il est alors davantage question de criminalité en col blanc (white collar crime) ou de délinquance des grandes sociétés (corporate crime).

Les conditions favorisant l'émergence d'entreprises criminelles sont la demande de biens et services illicites, l'insatisfaction de la demande de biens et services licites (en cas de pénurie par exemple), l'état du marché de l'emploi, l'état de l'encadrement normatif des activités économiques. Selon Lev Timofeev, le marché des stupéfiants montre que «de tous les modes possibles de régulation d'une branche d'activité imposition, nationalisation, interdiction -, l'interdiction est le moins productif. Interdire un marché ne signifie pas son anéantissement, mais revient à le placer sous le contrôle total des organisations criminelles et à attribuer à ces dernières la possibilité d'exercer une influence sur l'Etat et la société ${ }^{12}$.

Le crime organisé, en tant qu'élément de la société, est non seulement relié à l'économie, mais aussi à la sphère politique. On peut envisager l'hypothèse selon laquelle le crime organisé représente un phénomène social lorsqu'il ne s'agit pas seulement de la somme des organisations criminelles, mais d'un ensemble d'interactions générales entre milieux criminel, économique et politique dans un pays donné. C'est aujourd'hui le cas en Russie. 
L'organisation criminelle

D'un point de vue juridique, l'article 35 du code pénal de la Fédération de Russie distingue les groupes criminels (prestoupnaïa grouppa) et les associations criminelles (prestoupnoe soobchtchestvo), considérées comme des groupes organisés soudés, créés pour commettre des infractions graves ou particulièrement graves, ou comme l'union de groupes criminels. Cette définition imprécise fonde l'activité des services répressifs dans le domaine de la lutte contre le crime organisé.

Il est plus difficile de définir l'organisation criminelle d'un point de vue criminologique. Il convient d'abord d'exclure les organisations criminelles à orientation politique (par exemple les organisations politiques fascistes), ensuite d'écarter les organisations légales qui, dans le cadre de leur activité, recourent à des méthodes criminelles. Les organisations criminelles créées afin de tirer profit de la production et de la distribution de biens et de services illicites représentent notre objet d'étude. Ces organisations gagneraient à être désignées comme des entreprises criminelles. En tant qu'organisations sociales, elles sont plus proches de l'idéal-type d'une organisation professionnelle que de la famille, du groupe d'intérêt ou de la société. Comme toute organisation professionnelle, l'organisation criminelle peut comprendre un plus ou moins grand nombre de membres, mener son activité sur une base plus ou moins durable, diversifier plus ou moins son activité. Elle est organisée de manière plus ou moins complexe, selon des règles clairement fixées qu'elle cherche à faire respecter, garantit la sécurité des activités menées et tend à maximiser ses profits. Les organisations criminelles sont caractérisées par une stabilité et une capacité d'adaptation importantes, en vertu d'exigences sévères quant à la sélection des cadres, à la discipline, au recrutement des collaborateurs les plus jeunes, les plus forts et les plus motivés. Elles sont donc extrêmement compétitives.

En conséquence, les entreprises criminelles sont caractérisées par les propriétés suivantes :

- la stabilité et la durabilité de l'association

- la recherche d'un profit maximal

- la production et la redistribution de biens et de services

- l'exercice d'activités licites et illicites

- le fonctionnement hiérarchique et la claire délimitation des compétences

- l'usage de la corruption

- la tendance à l'appropriation monopolistique d'un secteur d'activité ou d'un territoire Par rapport aux autres organisations professionnelles, les organisations criminelles se caractérisent par un usage systématique de la corruption et par la production et la redistribution de biens et services illégaux.

Les acteurs du crime organisé en Russie 
Le crime organisé, tel qu'il se manifeste aujourd'hui, est profondément enraciné dans l'histoire russe. L'ensemble de ses activités est exercé par trois types d'acteurs : «les criminels traditionnels ", « les criminels économiques », « les nouveaux venus ».

Les acteurs traditionnels du crime organisé existaient déjà dans la Russie tsariste. Ils étaient insérés dans une organisation fortement hiérarchisée, dominée par les "voleurs", ou "voleurs dans la loi", selon la terminologie employée à partir des années 1930. Les catégories de criminels les plus respectées exerçaient des activités «techniques" spécialisées: pickpockets, cambrioleurs, escrocs, etc. Les auteurs de crimes de sang n'étaient pas respectés. Les associations criminelles régionales et locales possédaient leur propre caisse commune. Cette caisse était alimentée par les contributions de chacun, calculées selon un pourcentage relatif au montant du butin de chaque opération. Les fonds de la caisse commune étaient employés pour soutenir les détenus, dans certains cas leurs proches, et pour développer les activités criminelles de l'association. Les gardiens de la caisse commune étaient élus. Un langage spécifique, appelé fenia, était en vigueur au sein du milieu criminel et variait considérablement selon les régions. Des règles, désignées sous le terme de « loi », devaient être observées par ceux qui se considéraient comme des criminels authentiques et respectables : refus de collaborer avec les représentants de l'Etat, de prendre part à des organisations politiques, syndicales ou militaires, engagement à exercer une activité criminelle de manière permanente, nécessité de justifier d'un séjour durable en prison, mépris pour la richesse, prosélytisme. Les criminels «traditionnels » étaient caractérisés par une volonté explicite de se situer en dehors de la société. Les tatouages permettaient de connaître certains éléments du curriculum vitae d'un voleur.

Les «criminels économiques» sont étroitement liés à l'évolution de la société soviétique. Selon certains chercheurs, ils sont apparus lorsque l'économie souterraine s'est structurée en Union soviétique, au cours des années $1960^{13}$. Les tsekhoviki ${ }^{14}$, c'està-dire l'élite de la criminalité économique, complétaient l'économie légale en offrant des biens de consommation frappés par la pénurie. La criminalité économique s'est constituée comme ensemble stable et structuré d'interactions au début des années 1980. Elle a donné une impulsion au développement des relations d'affaires. Les teneviki ${ }^{15}$ recouraient à des pratiques illicites telles que la corruption, les abus, la falsification de documents, les atteintes à la propriété ou la spéculation. Dans ce milieu, les relations étaient fondées sur des liens de parenté, une appartenance territoriale commune, une connaissance de l'autre, l'existence de contacts privilégiés avec la nomenklatoura du Parti ou l'administration. Simultanément, les relations avec le milieu criminel étaient indispensables. En effet, les teneviki, souvent victimes d'extorsion ou d'autres infractions, devaient nécessairement s'adresser à des " autorités" de la pègre et contribuer à la caisse commune ${ }^{16}$. Cette contribution fut fixée à $10 \%$ des bénéfices dans les années 1970 , lors d'une réunion du milieu criminel ${ }^{17}$. $\mathrm{Au}$ cours des années 1980, les capitaux illicites ont pu être investis. Les dépôts bancaires et les investissements se sont substitués au traditionnel bas de laine. Les " criminels économiques » ont accumulé un important capital de relations au sein des élites dirigeantes et du milieu criminel et ont élaboré des schémas complexes de production clandestine, de commerce et d'écoulement des marchandises. La criminalité économique se définissait par l'exploitation des difficultés socio-économiques auxquelles était confronté le pays. 
A la fin des années 1980 et au début des années 1990, sont apparus des criminels d'un nouveau genre, les «nouveaux venus » ${ }^{18}$, dont les représentants ont pu être appelés «sportifs» ou plus fréquemment «bandits». Des jeunes, des sportifs (anciens ou en activité), des anciens de la guerre d'Afghanistan et d'anciens agents des services de sécurité, licenciés pour une raison ou pour une autre, se sont alors regroupés. Bien préparés physiquement, ils ne se distinguaient généralement pas par un passé judiciaire chargé. De nouvelles règles ont progressivement été élaborées. L'extorsion (le racket) est devenue la pratique criminelle la plus répandue. Les groupes n'hésitaient pas à agir avec cruauté, à employer des armes ou des explosifs. Ils ne reconnaissaient pas les règles traditionnelles du milieu et l'autorité des «voleurs dans la loi », ce qui a entraîné de nombreux conflits.

Il serait erroné de penser que les trois groupes d'acteurs décrits représentent isolément ce qui est appelé «crime organisé». C'est en fait le jeu de leurs interactions (coopération / concurrence) qui a conduit à la naissance du crime organisé à la fin des années 1980 (alors que, comme nous l'avons déjà observé, des organisations criminelles, dominées par les «voleurs dans la loi» existaient de longue date). L'adoption de la loi de l'URSS « sur la coopération » ${ }^{19}$ en mai 1988 favorisa l'émergence d'un milieu d'affaires et la légalisation de certaines activités menées par les criminels économiques et les organisations criminelles. Les «nouveaux venus ", dont les groupes sont caractérisés par la jeunesse, la souplesse et la rapidité d'adaptation, ont pris une part active dans ce processus économique. Les conflits d'intérêts et de règles ont d'abord entraîné des luttes impitoyables, mais par la suite, les différentes parties se sont retrouvées autour d'un même type symbiotique de criminalité. Les criminels « traditionnels » sont devenus de plus en plus tolérants à l'égard du monde des affaires, et ont de plus en plus recouru à l'établissement de contacts avec les institutions politiques et administratives. Certains tabous ont été levés. Les "nouveaux venus", quant à eux, ont progressivement reconnu l'autorité des "voleurs dans la loi » dans certains domaines, notamment dans l'organisation de la vie en détention. Les "criminels économiques" enfin sont devenus des hommes d'affaires exerçant leur activité dans des secteurs attractifs pour les deux autres groupes d'acteurs. Tous ces acteurs ont obligatoirement dû composer avec un quatrième groupe, auquel est intrinsèquement lié le développement du crime organisé en Russie : le milieu politicoadministratif.

En conséquence, les modes de résolution des conflits propres au milieu criminel ont progressivement imprégné les relations d'affaires. L'usage de la violence, du chantage, de la tromperie ou de la corruption, et l'emploi d'un certain argot n'ont plus été l'apanage exclusif de la pègre, mais également du milieu des affaires et de la politique. Dans la mesure où l'Etat éprouve des difficultés à réguler de nombreuses activités sociales, des règles informelles de comportement se sont substituées aux lois formelles, ineffectives ou lacunaires. Ces règles, parfois héritées des normes en vigueur chez les criminels traditionnels, agissent sur les comportements et représentent le fondement d'institutions sociales alternatives ${ }^{20}$. Le crime organisé mine, contourne et s'oppose aux formes étatiques du contrôle social ${ }^{21}$, tout en imposant ses propres formes de régulation ${ }^{22}$. Loin d'être un Etat dans l'Etat, le crime organisé remplit les niches que le contrôle étatique a laissé vacantes : fiscalité, justice ou arbitrage des contentieux par exemple.

De Leningrad à Saint-Petersbourg 
C'est durant les années 1970 et le début des années 1980 que le crime organisé a commencé à prendre sa forme actuelle. Compte tenu de la situation géographique de Leningrad, les opportunités offertes pour la contrebande ont toujours été particulièrement fortes. Durant les années 1970, les trafics portaient notamment sur les œuvres d'art, les antiquités et les biens de consommation courante (vêtements, appareils électroménagers). La contrebande d'antiquités et d'œuvres d'art requérait une "infrastructure criminelle »: un réseau ramifié d'« agents ", chargés notamment de collecter les informations et de choisir les victimes, était à l'œuvre à Leningrad. Les personnes âgées, les personnes seules, les alcooliques ou les émigrants sur le départ représentaient la majeure partie des victimes. Les méthodes employées - vol, extorsion, chantage ou escroquerie - pouvaient s'accompagner de violence.

De nombreuses « équipes » de fartsovchtchiki, c'est-à-dire de spéculateurs, étaient déjà en activité. Leur activité consistait à acheter et à revendre des biens de consommation courante. Pour accéder à des marchandises ou à des produits rares et sujets à une forte demande, il fallait soit les acheter en gros directement chez le producteur, soit "bloquer " les stocks dans les magasins, soit les acquérir auprès d'étrangers ou de Soviétiques ayant séjourné hors des frontières. La vente s'effectuait dans des marchés semi-légaux, situés non loin des grands magasins. A Leningrad, les arcades du Gostinii Dvor ${ }^{23}$ étaient par exemple bien connues pour abriter des vendeurs de parfum ou de produits de beauté, ainsi que des trafiquants de devises. Cette ville est d'ailleurs rapidement devenue l'un des centres vers lequel convergeaient les fartsovchtchiki de toute l'Union soviétique.

«Les bandes, si on peut les appeler ainsi, rassemblaient alors dix à vingt personnes. Elles s'occupaient en premier lieu de spéculation. Tous les vendeurs avaient déjà un protecteur, un "père ", comme on disait alors. Personne ne me touchait tant qu'on savait que je buvais des verres avec untel ou untel, considéré comme une "autorité ». La plupart des «autorités » de l'époque sont souvent devenues par la suite des figures notoires du crime organisé ${ }^{24}$.

A Leningrad, les bandes de spéculateurs exerçaient leur activité sur un territoire. L'« autorité », plus ou moins notoire, garantissait l'invulnérabilité du territoire, la protection des spéculateurs et se chargeait souvent de leur approvisionnement, en négociant avec les tsekhoviki, les producteurs clandestins, qui ont joué un rôle considérable dans le développement du crime organisé. La plupart des biens de consommation courante soi-disant étrangers, en particulier les vêtements "importés ", étaient alors produits à Leningrad et dans la région.

Durant les années 1970, le trafic de drogues a commencé à se développer. Les hippies soviétiques de l'époque, appelés "systèmes ", et les gitans s'occupaient généralement du transport et de la vente. La production s'effectuait dans les républiques soviétiques d'Asie centrale, dans l'extrême-Orient soviétique et dans quelques régions et républiques du sud de l'Union soviétique (Ukraine, territoire de Krasnodar, territoire de Stavropol). Un tel marché, même embryonnaire, ne pouvait de toute évidence fonctionner sans le concours du milieu criminel. Peu d'informations demeurent néanmoins disponibles sur ce point.

Jusqu'au milieu des années 1980, certaines bandes présentaient des modes de fonctionnement proches de ceux qu'on prêtera plus tard au crime organisé. Elles exploitaient les spécificités économiques du régime politique soviétique, notamment 
les pénuries et la planification. Leningrad n'a cependant jamais connu l'emprise d'un ou de plusieurs « voleurs dans la loi » ${ }^{25}$ sur les différentes bandes agissant en ville.

"A ma connaissance, la plupart des voleurs dans la loi dont on a parlé ici venaient d'ailleurs. Le plus souvent, ils étaient désignés au cours de réunions à Moscou afin de prendre en charge Piter ${ }^{26}$ et la région. Désignés par qui... on ne sait pas vraiment. C'est pas clair, comme si Piter échappait à tout ce système. C'est d'autant plus bizarre que dans des villes plus petites, comme Volkov, Tcherepovets ou Vologda, il y a des voleurs qui font autorité... Un moment, ici, il y en avait un, tonton Slava, il a été tué. C'était pas vraiment un voleur, c'était un polojenets ${ }^{27}$, mais ça, c'est un détail ; l'important, c'est qu'il faisait partie de tout ce système et qu'il a été tué ».

A la fin des années 1980 et au début des années 1990, dans le sillage du développement du secteur coopératif ${ }^{28}$, ont surgi à Leningrad des dizaines de groupes, composés de "nouveaux venus", instaurant et maintenant un contrôle sur un territoire donné : alentours de grands magasins, de marchés, de restaurants, d'hôtels, de cafés... Ces groupes obligeaient les vendeurs, les responsables des coopératives, puis les entrepreneurs en général à payer un impôt. Sur ces territoires, les acheteurs avaient également des chances d'être escroqués, lors d'une partie de bonneteau ou d'un échange de devises. Les groupes criminels veillaient à ce que le territoire reste en leur possession et à ce que la police "intervienne à temps » en cas d'embrouille ou d'arnaque.

De nombreuses activités sont alors devenues lucratives. Il s'agissait principalement d'activités exploitant les fortes distorsions entre l'offre et la demande de certains produits de grande consommation. A Saint-Petersbourg, le commerce d'automobiles volées, d'équipement bureautique (photocopie, télécommunication, informatique) et de «navettes " ${ }^{29}$ a été particulièrement rentable au début des années 1990. Ces trois activités, liées à la situation géographique et économique de Saint-Petersbourg, rendaient obligatoire la détention d'une ressource spécifique, l'existence de contacts étroits à l'étranger. Quant au commerce d'automobiles volées, les lieux de vente et le nom de l'organisation qui prend en charge cette activité ont toujours été notoires. Les voitures volées en Europe occidentale continuent de transiter par les PECO et aboutissent en Russie, souvent sur le ferry qui relie Kil et Saint-Petersbourg. Retrouver en Russie une voiture occidentale volée est fort difficile car la police des routes, l'un des services les plus corrompus du ministère de l'Intérieur de Russie, n'effectue aucune recherche sans perspective de rétribution.

«L'influence du crime organisé sur le secteur automobile est remarquable. D'une certaine manière, on peut dire que l'usine VAZ à Togliatti était contrôlée par des bandits jusqu'en 1997, c'est-à-dire avant qu'Anatoli Koulikov ${ }^{30}$ n'y mène une grande opération de nettoyage. Or que s'est-il passé ? La qualité de la production a baissé depuis! Sur le marché, tout le monde sait qu'une Jigouli ${ }^{31}$ d'occasion fabriquée en 1996 vaut mieux qu'une Jigouli neuve de $1999 »$.

Quant au commerce de matériel bureautique, il a souvent constitué l'étape initiale de l'enrichissement de nombreux hommes d'affaires russes. A la fin des années 1980 et au début des années 1990, ces marchandises étaient déficitaires et leurs prix bien plus élevés en Russie que sur le marché mondial. Une opération courante consistait à acheter à l'étranger du matériel d'occasion, l'importer comme pièces de rechange afin d'éviter les taxes et le vendre à Saint-Petersbourg à un prix élevé. Ce fut l'un des premiers moyens de s'enrichir rapidement, de manière semi-légale. 
Quant au "commerce de navettes » enfin, il s'agissait simplement de se rendre à l'étranger, d'acquérir en gros des biens de consommation courante, de les acheminer et de les revendre en Russie. Dans la mesure où les «navettes" partaient avec d'importantes sommes d'argent et rentraient en Russie avec une grande quantité de marchandises, elles devaient nécessairement chercher une protection. Comme l'Etat ne défend pas les intérêts des citoyens russes à l'étranger, les groupes criminels s'en sont rapidement chargés, en étant représentés dans les lieux d'achat afin d'assurer une protection contre paiement.

En dehors de ces activités qui, jusqu'au milieu des années 1990, profitaient des défauts du marché russe afin d'assurer la fourniture d'équipements sujets à une forte demande, les groupes criminels fournissaient simultanément des biens et des services illicites (prostitution, jeux, stupéfiants). Dans le domaine du jeu, ils possédaient des tripots, contrôlaient les stands de loterie dans la rue et ont commencé à ouvrir des casinos. Alors que, dans ce dernier cas, les impôts sont extrêmement élevés, et les licences et autorisations difficiles à obtenir, il existe à Saint-Petersbourg des dizaines de casinos et des centaines de salles de jeux avec des machines à sous. Ces établissements permettent en fait de blanchir facilement des capitaux et de monnayer les services rendus.

«C'est pas compliqué : t'invites la personne requise et tu lui fais gagner autant que tu le désires. C'est facile, propre, sans pots-de-vin. Quant à l'utilisation des casinos pour le blanchiment... faites une descente de police dans n'importe quel établissement et vous trouverez toujours des irrégularités. Il suffit d'avoir dans sa clientèle un conseiller du gouverneur ${ }^{32}$, quelqu'un de ce genre, et les ennuis sont écartés ».

Au début des années 1990, les associations (soobchtchestvo) criminelles " ethniques ", selon la terminologie russe, c'est-à-dire les bandes composées de ressortissants du Caucase ou d'Asie centrale, ont pris de l'ampleur à Saint-Petersbourg, en jouant notamment un rôle important dans le trafic de stupéfiants. D'une manière générale, la ville était alors partagée en sphères d'influence. Les conflits ne se résolvaient plus avec les poings, comme avant, mais avec des armes à feu. "Le milieu criminel de SaintPetersbourg ", tel qu'on le connaît aujourd'hui, s'est constitué à cette époque : quatre ou cinq puissantes associations de criminels, et des dizaines de groupes de moindre importance. Ces derniers, appelés bespredelchtchiki ${ }^{33}$, sont combattus par les premières, qui n'ont pas intérêt à ce que le désordre se développe sur leur territoire. Les associations les plus importantes ont pour point commun une activité initiale centrée sur le racket et la protection des entreprises situées sur leur territoire.

«Le racket, c'était surtout au départ. Aujourd'hui, il reste dans les groupes quelques types qui ne s'occupent que de ça. Ils suivent les affaires, résolvent les conflits, augmentent le nombre d'entreprises ou de commerces protégés ».

Dès le début des années 1990, l'activité du crime organisé s'avère déjà liée aux forces et faiblesses de l'Etat dans une société en transformation. L'absence, l'insuffisance ou les défauts de la régulation juridique et politique ont ouvert de nombreuses opportunités dans les domaines économique et social. Dans un tel contexte d'anomie, le crime organisé a fait preuve d'une capacité d'adaptation rapide et efficace. De nouveaux domaines d'activité ont pu être envisagés par les "nouveaux venus", capables d'utiliser des ressources économiques, financières et coercitives inédites.

La mise en œuvre du processus de privatisation, à partir de 1992, a ouvert des perspectives extraordinaires dès les années 1993-1994. En l'absence d'une régulation juridique claire, la privatisation a représenté de facto un mode d'appropriation des 
biens à bas prix par la nomenklatoura et les organisations criminelles. La violation des règles de déroulement des enchères et les menaces exercées sur les concurrents ont permis d'acheter ou de contrôler de nombreuses entreprises de la ville pour une bouchée de pain.

«La privatisation a offert aux intérêts privés des possibilités de profit qu'il était auparavant difficile d'envisager. A Petersbourg, l'appartenance de certaines grandes usines à des groupes criminels ne fait aucun doute. Les bandits ne se montrent certes pas dans l'usine et aucun racket n'y est effectué, mais ils orientent en revanche les choix liés à la production et à l'approvisionnement en matières premières ".

La protection des intérêts privés a progressivement obéi à des règles différentes. Alors qu'auparavant, les groupes criminels " couvraient " ${ }^{34}$ la plupart des commerces et des entreprises, les services répressifs sont devenus des concurrents et ont acquis une place accrue dans ce domaine. Des opérations de grande ampleur ont été menées contre des groupes réputés pour leur influence au niveau d'un quartier. L'arrestation de certaines figures emblématiques a entraîné une nouvelle lutte pour le partage et l'allocation des territoires. Les services répressifs se sont alors souvent substitués aux protecteurs précédents, informellement ou par l'intermédiaire d'entreprises de sécurité privée placées sous leur contrôle plus ou moins direct. Ils pouvaient signer des contrats avec des entreprises, s'accorder illégalement sur leur protection ou confier la prestation de services à une entreprise de sécurité privée fondée par d'anciens collègues. Des professionnels qualifiés issus des services répressifs ont quitté leur travail au profit du secteur de la sécurité privée.

«Avant, les gros bras des groupes criminels s'occupaient du racket. Ils écumaient la ville, un fusil mitrailleur à la main, se faisaient attraper et emprisonner. Au bout d'un moment, la police, ainsi que les autres services de sécurité, ont commencé à proposer leurs services aux entreprises privées, de manière légale ou non, qu'importe. Une sacrée concurrence : la moitié au moins des entreprises est aujourd'hui protégée par la police. D'où la nécessité pour les groupes criminels de fonder des entreprises légales, spécialisées dans la protection. On pouvait alors recruter des gros bras et continuer comme avant ».

Au milieu des années 1990, les activités criminelles les plus rentables sont devenues le trafic de stupéfiants, le marché du logement, le commerce de métaux, d'armes, la production et la vente de disques, vidéo et logiciels pirates dans un contexte de faible protection des droits d'auteurs, et la contrefaçon de vins et spiritueux. La privatisation des logements a conduit certains groupes criminels possédant des agences immobilières et des offices de notaires à repérer les propriétaires les plus vulnérables et à extorquer leurs biens. L'acquisition d'usines ou de friches industrielles est également devenue importante. Grâce à la corruption ou à l'usage de la force, il fut désormais possible d'acquérir des usines et de développer une nouvelle activité, en y ouvrant des clubs ou des restaurants par exemple. La mise en faillite artificielle d'une usine a fréquemment permis d'abandonner une activité productive au profit de l'ouverture d'un centre de loisirs ou d'affaires, beaucoup plus rentable.

Le commerce et la contrebande de métaux précieux ont également prospéré. De nombreux centres de stockage et de transport de métaux ont été fondés à SaintPetersbourg. L'origine de ces métaux était très souvent criminelle. Des réseaux se sont par exemple spécialisés dans le détournement de métaux non ferreux (tsvetnye), par exemple de cuivre (issu des canalisations) ou de bronze (provenant de monuments ou 
d'éléments décoratifs municipaux). Quant aux métaux rares (redkozemelnye), leur commerce rendait nécessaire l'existence de liens avec l'Oural, notamment la région de Sverdlovsk. Ils étaient par la suite exportés à l'aide de faux documents vers les pays baltes ou l'Europe occidentale. Des opérations similaires ont pu être menées avec l'essence ou le bois.

"A cette époque [vers 1994], il était très simple d'exporter illégalement des métaux de Russie. Les agents de l'administration ne s'en privaient pas! Les profits étaient mirobolants, compte tenu de la distorsion des prix entre le marché russe et, par exemple, le marché estonien. Des fortunes colossales ont été gagnées en deux-trois mois ».

Le commerce des armes, extrêmement rentable, s'est répandu. L'armée russe a parfois perdu le contrôle des stocks éloignés dans lesquels les armes sont entreposées. Beaucoup d'entrepôts se sont par ailleurs subitement trouvés en terre étrangère, désormais situés dans les anciennes républiques de l'Union soviétique. Les détournements se sont multipliés. Par ailleurs, le contrôle des armements s'est compliqué dans les zones de combat. Enfin, les groupes criminels ont consacré d'importants moyens à l'acquisition d'une influence dans les villes russes connues pour abriter de vastes centres de production d'armes (Toula, Ijevsk). La contrebande et la contrefaçon d'alcool se sont extrêmement développées. Un savoir-faire avait déjà été accumulé en 1985, lorsque Mikhaïl Gorbatchev, à peine arrivé au pouvoir, lança la « loi sèche » destinée à stopper la consommation d'alcool en Union soviétique. Depuis le début des années 1990, la production d'alcool, légale, est caractérisée par de faibles coûts de fabrication et souvent associée à des détournements et à de faibles exigences en matière de qualité. De grandes marques étrangères proviennent des alambics et des caves de Saint-Petersbourg et de la région.

Enfin, toujours à la même époque, l'exploitation du troc inter-entreprises, encouragé par le premier ministre Viktor Tchernomyrdine afin de régler l'épineux problème des impayés dans l'économie russe, a ouvert de considérables opportunités d'enrichissement. De longues chaînes d'entreprises reliées par des opérations de troc de marchandises ou de matières premières sont apparues, favorisant la falsification des documents comptables, la diminution de la valeur des marchandises et la dissimulation des profits et bénéfices.

" Notre gouvernement a lui-même conçu un système de troc merveilleux. L'Etat ne parvient pas à remplir ses obligations, en l'occurrence à régler ses dettes. Les entreprises se doivent de l'argent les unes aux autres d'abord parce que l'Etat leur en doit à toutes. Le système élaboré a permis de décharger l'Etat de ses obligations. Mais pour cela, il faut des managers qui puissent assurer un rôle d'intermédiaire entre les entreprises, ce qui n'est pas évident car ils subissent les pressions de chaque partie, désireuse de limiter au maximum le coût de l'opération ».

Il est frappant de constater à quel point l'Etat figure fréquemment dans les discours tenus par les représentants du crime organisé sur leur activité. Les représentants du milieu criminel présentent leur activité comme une conséquence de l'incapacité de l'Etat à jouer son rôle. Ils s'attribuent ainsi un rôle de "sauveur", d'acteur social « responsable».

"Je prends en charge ce que ne peut faire l'Etat: garantir le fonctionnement normal des affaires et la sécurité. C'est ça, mon business, et je ne m'y prends pas trop mal, crois-moi ». 
Ce discours peut éventuellement se traduire par un renversement normatif, employant les étiquettes de «criminel» et de "mafia» pour désigner un comportement moralement contestable et attribuer à l'«Etat» ou au «gouvernement»la responsabilité d'un problème social.

" [J'entends souvent dire que] plus une législation est mauvaise, plus elle avantage les criminels. Quelle sottise... Comment des lois de merde peuvent-elles avantager un criminel? Personne ne peut bien vivre dans une société instable. Ces lois avantagent uniquement ceux qui les écrivent, les bandits qui gouvernent ».

«Est-ce que la mafia existe? Bien sûr. Tu n'imagines pas les affaires auxquelles un poste officiel donne accès. A Petersbourg et ailleurs. Je connais des petites villes dans lesquelles l'administration municipale s'est octroyée la moitié des appartements. Tout le monde est de mèche : agents municipaux, policiers, députés, bandits. Voilà une vraie mafia».

"J'ai certes du pouvoir, mais il y a plus puissant que moi, notamment au sein du ministère de l'Intérieur, des services de sécurité ou des administrations en charge des privatisations. Ils occupent des fonctions officielles, mais ils se font encore plus d'argent que moi ».

Ces discours ont beau être motivés par un souci de présentation de soi sous un jour honorable, ils soulignent que, dans une période où les règles et les modes de légitimation sont instables, les représentants du crime organisé en Russie justifient leur activité en relation à celle de l'Etat, en tant que mode d'organisation de la société, et dénoncent l'usage du label de mafia en le retournant contre ceux qui, en les désignant ainsi, leur font porter la responsabilité des difficultés socio-économiques que traverse le pays.

Les entretiens avec les représentants des groupes criminels permettent de comprendre l'évolution des activités du crime organisé à Saint-Petersbourg et d'envisager les conclusions suivantes :

Les niches qui se sont formées lorsque l'Etat a perdu le contrôle d'une grande quantité d'activités sociales ont été occupées par d'autres groupes d'acteurs. Les contradictions et l'inapplication des lois russes favorisent la prédominance des règles informelles dans les pratiques économiques, la fiscalité étouffante incite à recourir à des pratiques illicites, le poids des administrations dans la vie économique encourage la corruption. L'imbrication du milieu des affaires et du milieu criminel fournit à ce dernier une influence certaine, y compris sur la production. Certains entrepreneurs, assurant parfois une part importante de la production dans un secteur donné, sont des délinquants notoires.

Dans de telles conditions, la criminalisation de la vie politique est inéluctable, au moins jusqu'à un certain niveau. Entrer en politique représente un moyen sûr d'obtenir l'immunité et de peser sur les décisions. Les pratiques criminelles (chantage, publication de documents compromettants, assassinats) marquent la vie politique russe. Les services de sécurité deviennent parfois de simples instruments dans ces règlements de compte.

Cependant, l'influence du crime organisé demeure strictement locale. Aucun groupe criminel russe ne peut prétendre avoir une activité nationale dans un pays si vaste, ou coordonner l'activité de différents groupes armés. En revanche, pour les groupes les plus puissants, l'existence de contacts dans certaines régions russes, riches en matière 
première ou productrices d'armes, et à l'étranger représente un signe de distinction au sein du milieu criminel.

Certaines activités d'ordinaire attribuées au crime organisé semblent inventées ou mythifiées. Aucune information ne permet d'attester la diffusion d'activités telles que l'envoi de bandits dans les conflits armés, le trafic d'organes, d'enfants, de matériaux radioactifs ou chimiques.

Il est donc urgent de dépolitiser et de désidéologiser les discours sur le crime organisé afin de distinguer les mythes de la réalité. Les besoins de connaissance sur cette forme de criminalité demeurent aujourd'hui considérables. Il est insupportable que la plupart des dirigeants politiques continuent d'affirmer que la « liquidation » du crime organisé est possible, que la fermeté peut payer et que le problème peut être résolu au moyen de mesures pénales et policières. Toute campagne de lutte anti-criminalité organisée, antidélinquance économique ou anti-corruption sert d'abord à neutraliser un adversaire ou un concurrent et à renouveler les agents administratifs occupant des postes stratégiques. Il importe de comprendre les facteurs socio-économiques qui expliquent le rôle du crime organisé dans la société russe aujourd'hui. Il s'agit à la fois de mieux réglementer les marchés, de mieux protéger les entreprises et de les inciter à respecter les règles légales, en diminuant les impôts et taxes auxquels elles sont assujetties, de mieux satisfaire les besoins de la population, et de donner aux jeunes la possibilité de s'épanouir. La conception et la mise en œuvre de la lutte contre le crime organisé doivent donc être complètement modifiées.

\section{Bibliographie}

Abadinsky H., Organized crime, Chicago, Nelson Hall, 1994 (quatrième édition).

Abramov E. (dir.), Griaznye dengi i zakon. Pravovye osnovy borby s legalizatseï prestoupnykh dokhodov (L'argent sale et la loi. Fondements juridiques de la lutte contre la légalisation des revenus criminels), Moscou, Infra-M, 1994.

Ageev L., Kazanskii fenomen : mif ili realnost? (Le phénomène de Kazan : mythe ou réalité ?), Kazan, 1991.

Aïdanian R., Gilinski I., «Founktsionalnaïa teoria organizatsii i organizovannaïa prestoupnost " (La théorie fonctionnaliste des organisations et le crime organisé), In Gilinski I., Organizovannaïa prestoupnost v Rossii : teoria i realnost (Le crime organisé en Russie: théorie et réalité), Saint-Petersbourg, Institout Sotsiologii Rossiiskoï Akademii Naouk, 1996. pp. 1-15.

Albanese J., "Organized crime : the Mafia mystique ", In Shelley J., Criminology: a contemporary handbook, Miami, Wadsworth, 1995.

Albini J., The American Mafia : genesis of a legend, New York, Appleton-Century-Crofts, 1971.

Barkan S., Criminology. A sociological understanding, Upper Saddle River, Prentice Hall, 1997.

Christie N., Crime control as industry, Londres, Routledge, 1993.

Cohen S., Folk devils and moral panics, St Alban, Paladin, 1973.

Dikselious M., Konstantinov A., Prestouny mir Rossii (Le monde criminel en Russie), Saint-Petersbourg, Bibliopolis, 1995.

Dolgova A., Diakov S. (dir.), Organizovannaïa prestoupnost (La criminalité organisée), Moscou, Iouriditcheskaïa literatoura, 1989. 
Dolgova A., Diakov S. (dir.), Organizovannaïa prestoupnost - 2 (La criminalité organisée - 2), Moscou, Kriminologitcheskaïa assotsiatsia, 1993.

Dolgova A., Diakov S. (dir.), Organizovannaïa prestoupnost - 3 (La criminalité organisée - 3), Moscou, Kriminologitcheskaïa assotsiatsia, 1996.

Dolgova A. (dir.), Organizovannaïa prestoupnost - 4 (La criminalité organisée - 4), Moscou, Kriminologitcheskaïa assotsiatsia, 1998.

Gilinski I., «O sistemnom podkhode k prestoupnosti » (Sur une approche systémique de la criminalité), Pravovedenie, $\mathrm{n}^{\circ}$ 5, 1981, pp. 49-56.

Gilinski I., Raska E., "O sistemnom podkhode k otkloniaiouchtchemousia povedeniou » (Sur une approche systémique des comportements déviants), Izvestia Akademii Naouk Estonskoï SSSR, vol. 30, n² 2, 1981, pp. 134-142.

Gilinski I., «Nekotorye voprosy metodologii kriminologitcheskikh issledovanii » (Quelques questions de méthodologie des recherches criminologiques), In Raska E., Teoretitcheskie problemy izoutchenia territorialnykh razlitchii $\mathrm{v}$ prestoupnosti (Problèmes théoriques de l'étude des différences territoriales dans le domaine de la criminalité), Tartu, Outchenye zapiski Tartouskovo ouniversiteta, 1988. pp. 102-110.

Gilinski I., Organizovannaïa prestoupnost v Rossii : teoria i realnost (Le crime organisé en Russie: théorie et réalité), Saint-Petersbourg, Institout Sotsiologii Rossiiskoï Akademii Naouk, 1996.

Gilinski I., "Organized crime in Russia : theory and practice ", Security Journal, $\mathrm{n}^{\circ}$ 9, 1997. pp. 165-169.

Gourov A., Professionalnaïa prestoupnost: prochloe i sovremennost (La criminalité professionnelle : histoire et actualité), Moscou, Iouriditcheskaïa Literatoura, 1990.

Gourov A., Krasnaïa mafia (La mafia rouge), Moscou, Samotsvet, 1995.

Gourov A., Riabinin V., Ispoved vora v zakone (Confession d'un voleur dans la loi), Moscou, Samotsvet, 1995.

Hulsman L., "Critical criminology and the concept of crime ", Contemporary crisis, $n^{\circ} 10,1986$, pp. 63-80.

Ioustitski V., "Organizovannaïa prestoupnost: smena paradigm » (La criminalité organisée: un changement de paradigmes), In Gilinski I. (dir.), Prestoupnost i kriminologia na roubeje vekov (La criminalité et la criminologie au tournant du siècle), Saint-Petersbourg, Institout Sotsiologii Rossiiskoï Akademii Naouk, 1999.

Kelly R. et al., Handbook of organized crime in the United States, Westport, Greenwood Press, 1994.

Khokhriakov G., Kriminologia (La criminologie), Moscou, Iourist, 1999.

Konstantinov A., Korroumpirovanny Peterbourg (Le Petersbourg de la corruption), Saint-Petersbourg, Folio Press, 1997.

Konstantinov A., Banditski Peterbourg (Le Petersbourg des bandits), Saint-Petersbourg, Folio Press, 1999.

Kostioukovski I, «Sotsialny kontrol i organizovannaïa prestoupnost » (Le contrôle social et la criminalité organisée), In Gilinski I., Sotsialny kontrol nad deviantnostiou v sovremennoï Rossii (Le contrôle social de la déviance dans la Russie contemporaine), Saint-Petersbourg, Institout Sotsiologii Rossiiskoï Akademii Naouk, 1998.

Koudriavtsev V., Eminov V. (dir.), Kriminologia (La criminologie), Moscou, Iourist, 1997. 
Louneev V., Prestoupnost XX veka. Mirovoï kriminologitcheskii analiz (La criminalité du vingtième siècle. Analyse criminologique mondiale), Moscou, Norma, 1997.

Milovanovic D., Postmodern criminology, New York, Garland, 1997.

Modestov N., Moskva banditskaïa (Le Moscou des bandits), Moscou, Tsentr-Poligraf, 1996.

Modestov N., Moskva banditskaïa - 2 (Le Moscou des bandits - 2), Moscou, TsentrPoligraf, 1997.

Oustinov V., Poniatie i kriminologitcheskaïa kharakteristika organizovannoï prestoupnosti (Concept et caractéristiques criminologiques de la criminalité organisée), Nijni Novgorod, Vyschaïa Chkola MVD RF, 1993.

Ovtchinski V., Strategia borby s mafieï (La stratégie de lutte contre la mafia), Moscou, SIMS, 1993.

Ovtchinski V., Eminov V, Iablokov N., Osnovy borby s organizovannoï prestoupnostiou (Les fondements de la lutte contre la criminalité organisée), Moscou, Infra-M, 1996.

Podlesskikh G., Terechonok A., Vory v zakone : brosok k vlasti (Les voleurs dans la loi : un bond vers le pouvoir), Moscou, Khoudojestvennaïa literatoura, 1994.

Razinkin V., Vory v zakone i prestoupnye klany (Les voleurs dans la loi et les clans criminels), Moscou, Kriminologitcheskaïa assotsiatsia, 1995.

Razinkin V., Tarabrin A., Elita prestounovo mira (L'élite du monde criminel), Moscou, Vetche, 1997.

Razzakov F., Bandity vremen kapitalizma (Les bandits du temps du capitalisme), Moscou, Eksmo, 1997.

Razzakov F., Bandity vremen sotsializma (Les bandits du temps du socialisme), Moscou, Eksmo, 1997.

Reid S., Crime and criminology, Madison, Brown and Benchmark, 1996 (huitième édition).

Siegel L., Criminology, St Paul, West Publishing Company, 1992.

Smith D., The Mafia mystique, Lanhamy, University Press of America, 1990.

Sumner C., The sociology of deviance : an obituary, New York, Continuum, 1994.

Tierney J., Criminology : theory and context, Londres, Prentice Hall, 1996.

Timofeev L., Narkobiznes : natchalnaïa teoria ekonomitcheskoï otrasli (Le commerce de la drogue : théorie élémentaire d'une branche économique), Moscou, RGGOu, 1998.

Topilskaïa E., Organizovannaïa prestoupnost (La criminalité organisée), SaintPetersbourg, Iouriditcheski Tsentr Press, 1999.

<!--SPIP--> 


\section{NOTES}

1. Ovtchinski, 1993, Gourov, 1995, Ovtchinski, Eminov, Iablokov, 1996, Topilskaïa, 1999, Louneev, 1997, Khokhriakov, 1999, Dolgova et Diakov, 1989, 1993, 1996, Dolgova 1998 (cf. bibliographie à la fin de l'article).

2. Voir les ouvrages de Konstantinov et de Dikselious.

3. Voir l'article de Gilles Favarel-Garrigues dans ce numéro.

4. De 1995 à 1997, la Fondation MacArthur a financé cette activité, dans le cadre de son programme intitulé « Organized crime centers in Russia ».

5. Hulsman, 1986, Tierney, 1996, Barkan, 1997.

6. Barkan, 1997, pp. 453-456.

7. Abadinsky, 1994, pp. 20-28.

8. Kelly et al. (ed.), 1994, pp. 78-88, Albanese, 1995, pp. 233-240.

9. Ioustitski, 1999, p. 46.

10. Koudriavtsev, V., Eminov, V., 1997, p. 258.

11. Ovtchinski, Eminov, Iablokov, 1996, p. 155.

12. Timofeev, 1998, p. 107.

13. Khokhriakov, 1999.

14. En russe, le terme tsekh désigne un atelier de production. Les tsekhoviki menaient une activité clandestine de production avec l'équipement dont ils disposaient au sein des entreprises.

15. Le terme tenevik désigne l'acteur économique qui agit au sein de l'économie " souterraine » (tenevaïa ekonomika).

16. Cette forme de protection sera par la suite désignée sous le terme de « toit » [krycha].

17. Ovtchinski, Eminov, Iablokov, 1996.

18. L'expression employée par l'auteur est littéralement « nouveaux criminels » (novye prestoupniki).

19. La loi sur la coopération a représenté l'une des premières mesures de privatisation de l'activité commerciale en Union soviétique.

20. Sur la pénétration des règles en vigueur au sein du milieu criminel dans la société soviétique et post-soviétique, voir l'article d'Anton Oleïnik dans ce numéro.

21. Topilskaïa, 1999, Khokhriakov, 1999.

22. Kostioukovski, 1998.

23. Centre commercial situé sur la perspective Nevski.

24. Conformément à nos engagements, noues ne fournissons aucune information sur nos interlocuteurs.

25. Il s'agit de ceux qui occupent le rang supérieur dans la hiérarchie de la pègre soviétique. Le titre est acquis lors d'une réunion de voleurs dans la loi, parfois appelée couronnement, au cours de laquelle les qualités du candidat - parcours professionnel, comportement en prison et en liberté, respect de la loi des voleurs - sont appréciées. Depuis la fin des années 1980, la tradition a souffert : le titre de voleur dans la loi est parfois acheté, la solidarité et l'homogénéité du groupe s'estompent.

26. Saint-Petersbourg.

27. Criminel notoire, disposant des droits et attributions des voleurs dans la loi sans avoir été « couronné ». 
28. Les coopératives furent les premières entreprises et les premiers commerces privés, dont le développement fut encouragé lors de la perestroïka. Elles ont souvent représenté la première étape du parcours professionnel des entrepreneurs russes qui ont développé leurs activités au début des années 1990.

29. « Navettes »est le nom donné à ceux qui, de manière pendulaire, achètent dans les pays voisins (dans le cas présent en Finlande ou dans les pays baltes) des marchandises en gros afin de les revendre en Russie. Le terme employé désigne la navette d'un métier à tisser.

30. Le ministre de l'Intérieur de l'époque.

31. C'est le nom de la voiture la plus populaire de Russie.

32. Dans les régions russes, le pouvoir exécutif est confié au gouverneur, élu au suffrage universel.

33. Littéralement, " ceux qui sont sans limite ", c'est-à-dire qui ne respectent pas les règles traditionnelles du milieu criminel.

34. En référence au terme de « krycha » (le toit).

INDEX

Mots-clés : mafias et crime organisé 